\title{
Parameter Adaptive Sliding Mode Trajectory Tracking Strategy with Initial Value Identification for the Swing in a Hydraulic Construction Robot
}

Jingwei hou ( $\sim$ houjw@jlu.edu.cn )

Jilin University

Dingxuan Zhao

Yanshan University

Zhuxin Zhang

Yanshan University

\section{Research Article}

Keywords: hydraulic servo, robust adaptive control, sliding mode, computer vision, construction robot

Posted Date: January 12th, 2022

DOI: https://doi.org/10.21203/rs.3.rs-1172316/v1

License: (c) (i) This work is licensed under a Creative Commons Attribution 4.0 International License.

Read Full License 


\section{Title page}

\section{Parameter Adaptive Sliding Mode Trajectory Tracking Strategy with Initial Value Identification for the Swing in a Hydraulic Construction Robot}

Jing-Wei Hou, born in 1978, is currently a PhD at School of mechanical and aerospace engineering, Jilin University, China. He received his $\mathrm{PhD}$ degree in Jilin University, China, in 2008. His research interests include engineering robot and hydraulic servo control.

Tel: +86-13504316084; E-mail: houjw@jlu.edu.cn

Ding-Xuan Zhao, born in 1965, is currently a professor at School of mechanical engineering YanShan University, China. He received his PhD degree in Jilin University, China, in 1992.

E-mail: zdx@ysu.edu.cn

Zhu-Xin Zhang, born in 1967, is currently a professor at School of mechanical engineering YanShan University, China. He received his $\mathrm{PhD}$ degree in Jilin University, China, in 2007.

E-mail: zzx@ysu.edu.cn

Corresponding author: Jing-Wei Hou E-mail: houjw@jlu.edu.cn 


\title{
Parameter Adaptive Sliding Mode Trajectory Tracking Strategy with Initial Value Identification for the Swing in a Hydraulic Construction Robot
}

\author{
Jing-Wei Hou' ${ }^{1}$ Ding-Xuan Zhao ${ }^{2} \cdot{\text { Zhu-Xin } \text { Zhang }^{2}}^{2}$
}

Received June xx, 2021; revised February xx, 201x; accepted March xx, 201x

(C) Scientific Reports and Springer-Verlag Berlin Heidelberg 2017

\begin{abstract}
'Abstract: A novel trajectory tracking strategy is developed for a double actuated swing in a hydraulic construction robot. Specifically, a nonlinear hydraulic dynamics model of a double actuated swing is established, and a robust adaptive control strategy is designed to enhance the trajectory tracking performance. When an object is grabbed and unloaded, the inertia of a swing considerably changes, and the performance of the estimation algorithm is generally inadequate. Thus, it is necessary to establish an algorithm to identify the initial value of the moment of inertia of the object. To this end, this paper proposes a novel initial value identification algorithm based on a two-DOF robot gravity force identification method combined with computer vision information. The performance of the identification algorithm is enhanced. Simulations and experiments are performed to verify the effect of the novel control scheme.
\end{abstract}

Keywords: hydraulic servo; robust adaptive control; sliding mode; computer vision; construction robot

\section{Introduction}

Due to the risks associated with the presence of operating errors and dynamic changes in the environment,

$\bowtie$ Jing-Wei Hou

houjw@jlu.edu.cn

1 School of mechanical and aerospace engineering, Jilin University, Changchun 130022, China

2 School of mechanical engineering YanShan University, Qinghuangdao 066004, China the development of fully automated systems for robotic construction machines or construction robots is limited, although automatic control can help achieve a high precision and efficiency for many kinds of machines [1,2]. To promote the operation of robotic construction machines, it is necessary to enhance the environmental perception and implement intelligent assistance with automatic control for novice operators [3-5]. By optimizing the proportional integral derivative (PID) controller, Feng [6] proposed an improved ant colony optimization algorithm (IACO) to increase the tracking accuracy of hydraulic systems. Moreover [7], a robust controller was designed using the $\mu$-synthesis method to ensure the stability and performance of a hydraulic excavator.

Zhao et al. developed a construction robot system with physical human-robot interaction (PHRI), which could perform tasks in earthquake disaster sites [8,9]. In such systems, as the end effector operation may be dangerous to the operator, a novel force strategy associated with a master-slave control schematic is usually adopted.

Most engineering robot systems are actuated through hydraulic servo systems that suffer from disturbances and uncertainties. To attain high-performance trajectory tracking control in uncertain nonlinear systems, the robust adaptive control method [10] has been widely used 
because of its flexibility and robustness. For example [11], a fuzzy logic controller was designed to track the trajectory of an industrial robot with 2 degrees of freedom. Three particle swarm optimization algorithms with different cost functions were used to optimize the controller parameters. The fuzzy logic in the algorithm decreased the complexity of mathematical modelling, which is commonly a complex and time-consuming process. However, owing to the lack of comprehensive analyses, the algorithm lacked generality in multiple situations, even though satisfactory results were obtained in the specific experiments. Among such strategies, adaptive sliding mode control is a valuable control method [13,14].

Adaptive sliding mode control is a new control strategy for nonlinear systems. This method combines the advantages of adaptive control and sliding mode control by introducing adaptive estimation into the sliding mode controller. According to the information uncertainty determined using the adaptive controller, the sliding mode controller can be adjusted to decrease the system uncertainty and conservativeness of the sliding mode control. In this manner, the system can maintain the robustness of sliding mode control to external disturbances and unmodelled dynamics, and the adaptive control strategy can help overcome the limitations of sliding mode control [15-18]. To suppress the motion disturbance of the actuator, a nonlinear robust dual-loop control scheme was proposed [19]. In addition to considering the motion disturbance of the actuator, the nonlinear characteristics and friction problems of the EHLS were considered. Furthermore, a continuous control set model predictive speed control (CCS-MPSC) based on the fast terminal cost index (FTCI) was proposed [20] to enhance the system tracking performance.

Although sliding mode control is highly robust, it is based on the precise mathematical model of the object [21-24]. The "rough" mathematical model of the system must be predicted before the system sliding mode surface parameters and controller parameters can be determined. For nonlinear systems, the dynamics of the nonlinear function of the system must be identified before designing the sliding mode controller. The method of robot parameter identification to obtain accurate model parameters is of significance in the design of robot controllers including sliding mode controllers. To address the uncertain load disturbances of a hydraulic Stewart manipulator, sliding mode control based on the discontinuous projection adaptive law was developed to enhance the tracking performance [25-27]. J. B Cao et al. used neural networks to adjust the switching gain online and realize system identification and parameter prediction. Moreover, the authors designed a neural network sliding mode controller for hybrid electric vehicles [28]. Using the Szász-Mirakyan operator as the basic function and by adjusting the polynomial coefficients through the adaptive law determined through stability analyses, a robust adaptive controller was designed to enhance the impedance control of a robot manipulator [29]. Other common methods to enhance the estimation performance include the use of neuro-fuzzy model auxiliary filters and time-varying parameters [30-34].

A satisfactory trajectory tracking controller must ensure that a robot can perform precise trajectory tracking. To this end, the dynamic characteristics of the robot must be considered [35-38]. To design the controller for a swing when a robot carries an object and increase the trajectory tracking accuracy, it is necessary to design a robust adaptive controller to address the uncertainty of the parameters. For a hydraulic cylinder system, the load equivalent mass is the most important element in controller design. However, for a swing, because the load mass is large and changes in real time according to the working conditions, the equivalent mass cannot be accurately obtained. Nevertheless, it can be assumed that the load does not change during the carrying process. Based on the inverse dynamic analysis of the robot arm and boom cylinders, the initial value of the equivalent mass can be obtained through system identification [39]. Subsequently, relatively accurate initial values of the controller parameters can be obtained, and the convergence and trajectory tracking accuracy of the controller can be enhanced. These aspects are the focus of 
this article.

The paper is organized as follows: The background and related research are described in Section 1. Section 2 presents the system overview of the teleoperation system and problem description. Section 3 describes the sliding mode adaptive robust controller for the swing. The method to identify the system initial value is described in Section 4. The simulation experiment is described in Section 5. Section 6 describes the experiment performed to compare the performance of three controllers carrying objects between two points.

\section{System and Problem Description}

\subsection{System description}

The proposed teleoperation system consists of two parts: a pair of hydraulic PHRIs as the master and an engineering robot reconstructed from a hydraulic excavator as the slave, as shown in Figure 1. Moreover, the system includes a control PC for each part.

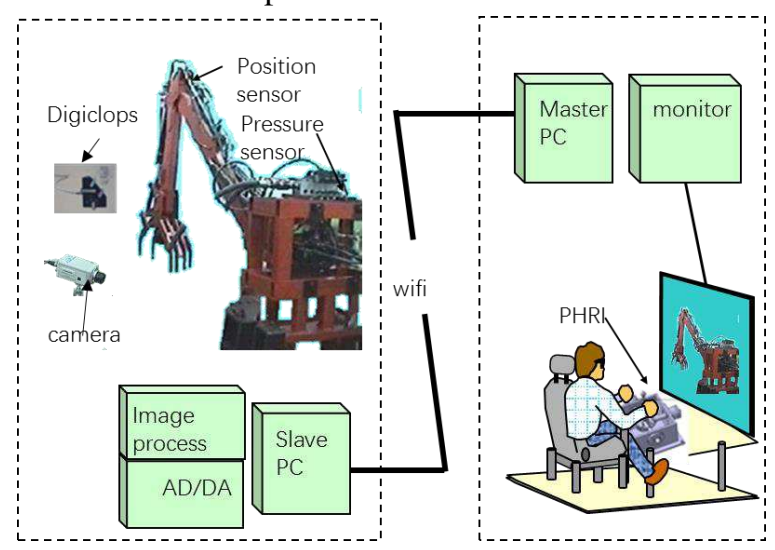

Figure 1 Teleoperation system

The four asymmetric hydraulic cylinders are driven by servo valves. The working pressure can be measured using pressure sensors installed in the rod and rod-less cavities of the hydraulic cylinder. The displacement of the working device can be determined using a displacement sensor installed outside the hydraulic cylinder.

The system uses cameras for monitoring. However, considering the limitations of the camera viewing angle and clarity, a 3D vision device is used to construct a virtual scene and the work object. The general shape of the object can be determined through the 3D camera, although the volume and shape cannot be accurately identified due to the low camera resolution.

Figure 2 shows the actuation system of the swing. The double-cylinder actuated swing occupies a small space and does not need to drive the heavy chassis. The production cost of the structure increases because of the use of the double cylinders. However, because multi-point transmission and effective force transmission can be realized by the hydraulic system, this design exhibits a low energy consumption and compact structure.

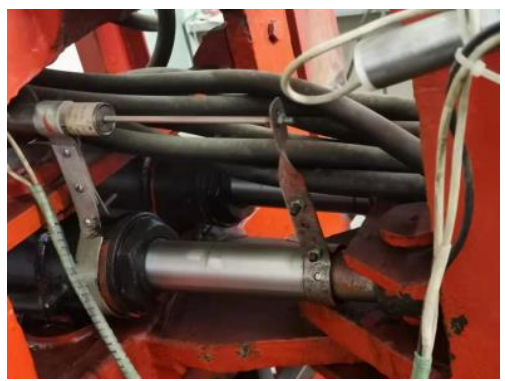

Figure 2 Swing DOF in the construction robot

\subsection{Problem description}

The moment of inertia parameter of the swing varies considerably when the work object is carried and unloaded. However, the parameter has a constant value during one procedure. This article designs a sliding adaptive robust trajectory tracking controller for the swing of a hydraulic construction robot. However, the strategy suffers from a low convergence speed when the moment of inertia parameter varies. Therefore, a moment of inertia identification algorithm is used.

The main problem can be divided into the following subproblems.

(1) According to the dynamic characteristics of the robot, the gravity compensation algorithm is used to determine the weight of the object according to the information provided by the arm and boom force sensors. The shape and size information of the working object is obtained through computer vision. These data points are combined with the weight information to determine the inertia of the object relative to the swing.

(2) To effectively suppress the uncertainties and disturbances, a sliding mode adaptive controller is designed for the hydraulic servo system of the swing. By combining the control strategy and initial value obtained by determining the moment of inertia, the parameter convergence and trajectory tracking 
performance can be enhanced.

\section{Sliding adaptive robust controller for the swing}

\subsection{Mathematical model of the hydraulic system}

The dynamic model for the hydraulic system shown in Figure 2 is

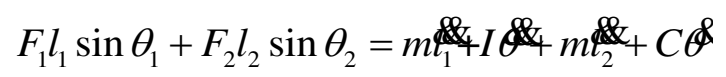

where $\mathrm{m}$ is the mass of the cylinder rod, $\mathrm{I}$ is the equivalent mass of the robotic arm and the cylinders, $F_{1}$ and $\mathrm{F}_{2}$ denote the load forces of the cylinders, and $\mathrm{C}$ is the damping coefficient.

In this case,

$$
\begin{aligned}
& \left(P_{1} A_{1}-P_{2} A_{2}\right) l_{1} \sin \theta_{1}+\left(P_{1} A_{2}-P_{2} A_{1}\right) l_{2} \sin \theta_{2} \\
& =m+I \&
\end{aligned}
$$

where $A_{1}$ and $A_{2}$ are the areas of the two sides of the piston, $\mathrm{C}$ is the coefficient of viscous friction, and $\mathrm{P}_{1}$ and $\mathrm{P}_{2}$ denote the pressures in the two chambers of the cylinder.

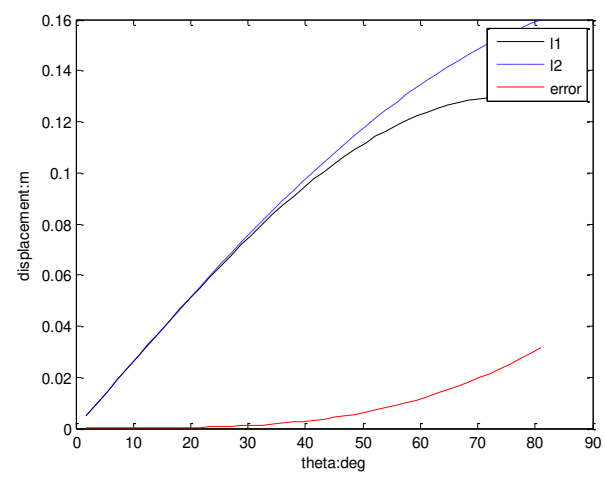

Figure $3 l_{1}$ and $l_{2}$ when the object is carried

(kinematics model of the double actuated swing)

The kinematics of the swing during the carrying process are derived in the Supplementary file. The result calculated using the MATLAB program is shown in Figure 3. The lengths $1_{1}$ and $l_{2}$ are small and exhibit similar amplitudes when the swing DOF varies from the centre position to the $60^{\circ}$ position.

$\mathrm{A}_{1} l_{1} \sin \theta_{1}+\mathrm{A}_{2} l_{2} \sin \theta_{2} \approx \mathrm{A}_{2} l_{1} \sin \theta_{1}+\mathrm{A}_{1} l_{2} \sin \theta_{2} \approx \mathrm{A}_{\mathrm{s}}$
$\Delta l_{1} \approx \Delta l_{2} \approx y$,

Subsequently, (2) can be expressed as

$$
\left(\mathrm{P}_{1}-\mathrm{P}_{2}\right) A_{s}=2 m I \& C \theta^{\&}
$$

According to Eq. (3), considering leakage and compressibility, the dynamics of the cylinder oil flow can be expressed as [17]

$$
\begin{aligned}
& \mathrm{V}_{1} \mathrm{P}_{1} / \beta_{e}=-A_{s} x_{l}+Q_{1}-C_{t}\left(P_{1}-P_{2}\right) \\
& \mathrm{V}_{2} \mathrm{P}_{2} / \beta_{e}=A_{s} x_{l}-Q_{2}+C_{t}\left(P_{1}-P_{2}\right)
\end{aligned}
$$

where $C_{t}$ is the internal leakage coefficient, $V_{1}$ and $V_{2}$ denote the total fluid volumes of the hydraulic cylinders, $\beta_{\mathrm{e}}$ is the bulk modulus of the fluid, and $\mathrm{Q}_{1}$ and $\mathrm{Q}_{2}$ are the fluid flow rates of the cylinders. The displacement of the spool valve, $x_{\mathrm{v}}$, is

$$
\begin{aligned}
& Q_{1}=k_{q} x_{v} \sqrt{\Delta P_{1}} \\
& Q_{2}=k_{q} x_{v} \sqrt{\Delta P_{2}}
\end{aligned}
$$

where $\mathrm{k}_{\mathrm{q}}$ is the flow gain coefficient of the servo valve.

The state variables are defined as

$$
\mathbf{x}=\left[\begin{array}{llll}
x_{1} & x_{2} & x_{3} & x_{4}
\end{array}\right]=\left[\begin{array}{llll}
y & \& & P_{1} & P_{2}
\end{array}\right]
$$

The dynamics are expressed in a state-space form as

$$
\begin{aligned}
& \&=x_{2} \\
& \&=\frac{1}{\mathrm{M}}\left[\left(x_{3}-x_{4}\right) A_{s}-B x_{2}-d\right] \\
& \&=\frac{\beta_{e}}{\mathrm{~V}_{0}}\left(-A_{s} y+k_{q} k_{a} \sqrt{\Delta P_{1}} u-C_{t}\left(x_{3}-x_{4}\right)\right) \\
& \&=\frac{\beta_{e}}{\mathrm{~V}_{0}}\left(A_{s} y-k_{q} k_{a} \sqrt{\Delta P_{2}} u+C_{t}\left(x_{3}-x_{4}\right)\right)
\end{aligned}
$$

where $\mathrm{M}$ is the equivalent load mass obtained from Eq. (3), $V_{0}$ is the total fluid volume of the two cylinders, and $\mathrm{A}_{\mathrm{s}}$ is the total area of the cylinders.

The time derivative of the second expression in (7) is

$$
\frac{1}{\mathrm{M}}\left[\left(\underset{3}{\&}-A_{s}-B \underset{2}{\&}-d\right]\right.
$$

In (8), the difference in the third and fourth terms is 
$\underset{3}{\&}=\frac{\mathrm{V}_{1}}{\beta_{e}}\left(-2 A_{s} y+2 k_{q} k_{a} \sqrt{\Delta P_{1}} u-2 C_{t}\left(x_{3}-x_{4}\right)\right)(9)$

Thus,

$\frac{-2 A_{s}^{2} x_{l} \beta_{e}}{M V_{0}}+\frac{A_{s} k_{q} k_{a}\left(\sqrt{\Delta P_{1}}-\sqrt{\Delta P_{2}}\right) \beta_{e}}{M V_{0}} u$

$-\frac{2 A_{s} \beta_{e} C_{t}}{M V_{0}}\left(x_{3}-x_{4}\right)-\frac{B}{M} \&-\frac{d}{M}$

\subsection{Controller design}

The following unknown parameter set is defined:

$\sigma_{1}=\frac{-2 \beta_{e} A_{s}^{2}}{M V_{0}}, \sigma_{2}=\frac{-2 \beta_{e} A_{s} C_{t}}{M V_{0}}, \sigma_{3}=\frac{B}{M}, \sigma_{4}=\frac{1}{M}$, $\sigma_{5}=\frac{\beta_{e}}{M V_{0}} k_{q} k_{a}$

The state-space Eq. (10) is linearly parameterized as [40]

$$
\begin{aligned}
& f=\sigma_{1} x_{2}+\sigma_{2}\left(x_{3}-x_{4}\right)+\sigma_{3} \&+\sigma_{4} d^{\&} \\
& g=\sigma_{5}\left(\sqrt{\Delta P_{1}}+\sqrt{\Delta P_{2}}\right)
\end{aligned}
$$

Function $f$ can be expressed as $f=\hat{f}+\Delta f$, where

$\hat{f}$ is the nominal part, and $\Delta f$ is the uncertain part, bounded as

$$
\begin{aligned}
& |\Delta f(\mathbf{x}, t)| \leq F(\mathbf{x}, t) \\
& \hat{f}=\hat{\sigma}_{1} x_{2}+\hat{\sigma}_{2}\left(x_{3}-x_{4}\right)+\hat{\sigma}_{3} \&+\hat{\sigma}_{4} \&
\end{aligned}
$$

where $\mathrm{F}$ is a known function.

The control gain $g$ is confined to a certain constant range.

$0<\beta_{\min }<g<\beta_{\max }$

The estimated value of $\mathrm{g}$ is

$$
\hat{g}=\hat{\sigma}_{5}\left(\sqrt{\Delta P_{1}}+\sqrt{\Delta P_{2}}\right)
$$

The control objective is to ensure that $x_{\mathrm{L}}$ asymptotically tracks the desired trajectory $x_{\mathrm{Ld}}$.

The tracking error is defined as

$$
y /=y-y_{d}
$$

The sliding mode surface is defined as

$$
s=\left(\frac{d}{d t}+\lambda\right)^{2} \quad f / \sigma=2 \lambda+\lambda^{2} \varphi /
$$

Differentiating s with respect to $t$ yields

$$
\begin{aligned}
& +2 \lambda+\lambda^{2} \phi r \\
& =f+g u-2 \lambda+\lambda^{2} \phi /
\end{aligned}
$$

The Lyapunov function candidate is defined as

$$
V(s)=\frac{1}{2} s^{2}
$$

Thus,

$$
\begin{aligned}
& I_{S} \&=s\left\{\sigma_{1} x_{2}+\sigma_{2}\left(x_{3}-x_{4}\right)+\sigma_{3} \&+\sigma_{4} \&\right. \\
& +\left[\sigma_{5}\left(\sqrt{\Delta P_{1}}+\sqrt{\Delta P_{2}}\right)\right] u-2 \lambda+\lambda^{2} \varphi \rho^{2}+2 \lambda
\end{aligned}
$$

The control input $u$ is

$$
\begin{aligned}
& u=\frac{1}{\hat{g}}(\hat{u}-v) \\
& \hat{u}=-\hat{f}+2 \lambda-\lambda^{2} \\
& v=\operatorname{ksat}\left(\frac{s}{\phi}\right)
\end{aligned}
$$

where $\mathrm{f}$ is the boundary layer, $\mathrm{k}$ is the control gain, and $\operatorname{sat}(\Delta)$ is the saturation function, defined as

$$
\operatorname{sat}(\Delta)=\left\{\begin{array}{c}
1, \Delta>1, \\
\Delta,|\Delta|<1, \\
-1, \Delta<-1,
\end{array}\right.
$$

To obtain the adaptation parameters, the following Lyapunov function is defined:

$$
V_{1}(s)=\frac{1}{2} s^{2}+\frac{1}{2} \sum_{i=1}^{5} \gamma_{i} \hat{\sigma}_{i}^{2}
$$

where $\hat{\sigma}_{i}$ is the estimated value of $\sigma_{i}$.

The estimation error is

$\delta \sigma_{i}=\sigma_{i}-\hat{\sigma}_{i}$ 
The time derivative of $\mathrm{V}_{1}$ is

$I_{1}^{\&}=s \& \sum_{i=1}^{5} \gamma_{i} \hat{\sigma}_{i} \delta_{i}^{\&}=-\hat{\sigma}_{1}\left[s \sigma_{1} x_{2}-\gamma_{1} \sigma_{1}\right]+$

$\hat{\sigma}_{2}\left[s\left(x_{3}-x_{4}\right)-\gamma_{2} \& \delta_{2}\right]+\hat{\sigma}_{3}\left(\&-\gamma_{3} \delta_{3}\right)$

$+\hat{\sigma}_{4}\left(\mathrm{~s} d+\gamma_{4} \& \sigma_{4}\right)\left[\sigma_{5}\left(\sqrt{\Delta P_{1}}+\sqrt{\Delta P_{2}}\right) u-\gamma_{5} \dot{\sigma}_{5}\right]$

$-k s a t\left(\frac{s}{\phi}\right) s+\sigma_{4} d s$

To ensure that $\mathbb{L}_{1}<0$, the adaptation laws are set as

$\stackrel{\&}{\&}=\operatorname{Pr} o j_{\hat{\sigma}_{1}}\left(-\frac{s \sigma_{1} x_{2}}{\gamma_{1}}\right)$

$\delta_{2}=\operatorname{Pr} o j_{\hat{\sigma}_{2}}\left(-\frac{s\left(x_{3}-x_{4}\right)}{\gamma_{2}}\right)$

$\delta_{3}^{\&}=\operatorname{Pr} o j_{\hat{\sigma}_{3}}\left(\frac{\hat{\sigma}_{3} \&}{\gamma_{3}}\right)$

$\delta_{4}=\operatorname{Pr} o j_{\hat{\sigma}_{4}}\left(-\frac{s d}{\gamma_{4}}\right)$

$\mathcal{\&}_{5}=\operatorname{Pr} \operatorname{oj}_{\hat{\sigma}_{5}}\left(\frac{\sigma_{5}\left(\sqrt{\Delta P_{1}}+\sqrt{\Delta P_{2}}\right) u s}{\gamma_{5}}\right)$

Function Proj() is a discontinuous projection, defined as

$\left.\operatorname{Pr}{o j_{\hat{\sigma}_{\mathrm{i}}}} \bullet\right)=\left\{\begin{array}{l}0 \text { if } \hat{\sigma}_{\mathrm{i}}=\sigma_{\mathrm{imax}} \& \bullet>0, \\ 0 \text { if } \hat{\sigma}_{\mathrm{i}}=\sigma_{\mathrm{imin}} \& \bullet<0, \\ \bullet \text { otherwise }\end{array}\right.$

For any adaptation function, the projection mapping ensures that

$\hat{\sigma}_{\mathrm{i}} \in\left\{\hat{\sigma}_{\mathrm{i}}: \sigma_{\mathrm{imin}} \leq \hat{\sigma}_{\mathrm{i}} \leq \sigma_{\mathrm{imax}}\right\}$

$\hat{\sigma}_{\mathrm{i}}\left[\gamma_{\mathrm{i}} \operatorname{proj}_{\hat{\sigma}_{\mathrm{i}}}\left(\gamma_{\mathrm{i}}^{-1} \Delta_{i}\right)-\Delta_{i}\right] \leq 0$

Controller (20) and adaptation laws (26) ensure that the tracking errors asymptotically converge to zero, i.e., $\ell(t) \rightarrow 0$ as time $t \rightarrow \infty$.

\section{Identification of the moment of inertia}

The swing DOF when an object is being carried pertains to a robot arm system with constant parameters that cannot be identified offline. As described in Section 3, the main parameters in (10) are dependent on the equivalent mass $M$. The equivalent mass $M$ is composed of the mass of the swing hydraulic cylinder push rod and moment of inertia I. Therefore, I is determined as

$$
I=I_{a}+I_{b}+I_{o}
$$

where $I_{a}$ is the moment of inertia of the arm and folk glove, and $\mathrm{I}_{\mathrm{b}}$ is the moment of inertia of the linkage system with the swing and boom. $I_{a}$ and $I_{b}$ can be accurately obtained through offline identification. $\mathrm{I}_{\mathrm{o}}$ is the moment of inertia of the object being carried, which varies in a large range and cannot be accurately calculated offline. A new method is proposed that combines the stereo vision system and hydraulic cylinder information of the boom and arm to obtain $\mathrm{I}_{\mathrm{o}}$.

The density and approximate shape and volume of the work object can be derived as

$$
I_{o}=\int \rho \mathrm{V}
$$

However, the shape and volume obtained by the vision system are considerably different from those of the work object, and thus, the direct use of these parameters may lead to a low estimation accuracy. The force obtained by the force sensor is highly accurate. If the mass of the work object can be obtained considering the force of the arm and boom, the moment of inertia can be corrected to obtain more accurate moment of inertia parameters.

The dynamic model for a system composed of booms, arms and folkgloves is [29]

$$
\begin{array}{r}
{\left[\begin{array}{c}
\tau_{b} \\
\tau_{a}
\end{array}\right]=\left[\begin{array}{cc}
m_{b} l_{g b} C_{b}+m_{a} l_{b} & m_{a} l_{g a} C_{a} \\
0 & m_{a} l_{g a} C_{a}
\end{array}\right]\left[\begin{array}{c}
C \theta_{b} \\
C \theta_{b a}
\end{array}\right] \mathbf{g}} \\
-\left[\begin{array}{cc}
m_{b} l_{g b} S_{b} & m_{a} l_{g a} S_{a} \\
0 & m_{a} l_{g a} S_{a}
\end{array}\right]\left[\begin{array}{l}
S \theta_{b} \\
S \theta_{b a}
\end{array}\right] \mathbf{g}
\end{array}
$$




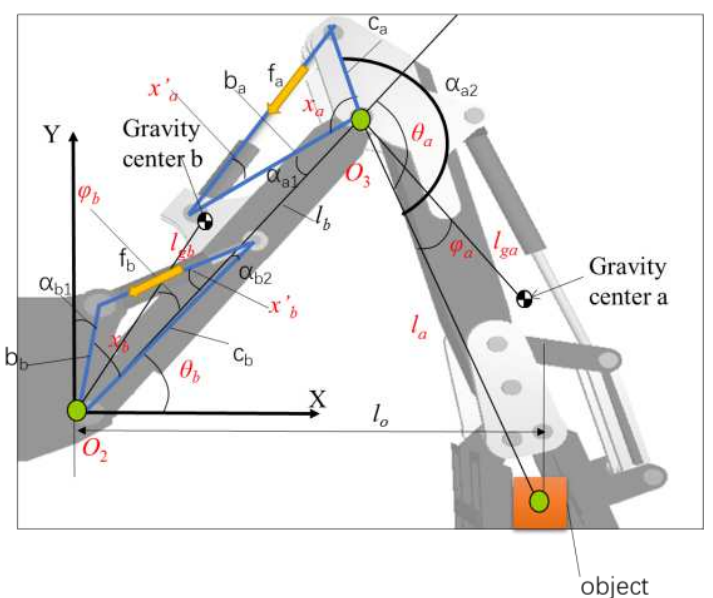

Figure 4 Boom and arm parameter

where $\tau_{\mathrm{a}}$ and $\tau_{\mathrm{b}}$ denote the torques of the arm and boom, respectively, as shown in Figure 4.

$$
\begin{aligned}
& C_{b}=\cos \varphi_{b}, C_{a}=\cos \varphi_{a}, S_{b}=\sin \varphi_{b}, S_{a}=\sin \varphi_{a} \\
& C \theta_{b}=\cos \theta_{b}, C \theta_{b a}=\cos \left(\theta_{b}-\theta_{a}\right), \quad S \theta_{b}=\sin \theta_{b}, \\
& S \theta_{b a}=\sin \left(\theta_{b}-\theta_{a}\right)
\end{aligned}
$$

The boom angle $\theta_{b}$ is

$$
\theta_{b}=x_{b}-\alpha_{b 1}-\alpha_{b 2}
$$

The arm angle $\theta_{a}$ is

$$
\theta_{a}=x_{a}-\pi+\alpha_{a 1}+\alpha_{a 2}
$$

The boom push force is

$$
f_{b}=\frac{\tau_{b}}{c_{b} \sin x_{b}^{\prime}}
$$

The arm push force is

$$
f_{a}=\frac{\tau_{a}}{c_{a} \sin \left(x_{a}+x_{a}^{\prime}\right)}
$$

When the object is carried,

$$
\begin{gathered}
{\left[\begin{array}{c}
\tau_{b} \\
\tau_{a}
\end{array}\right]=\left[\begin{array}{cc}
m_{b} l_{g b} C_{b}+m_{a} l_{b}+m_{o} l_{o} & \left(m_{a} l_{g a}+m_{o} l_{o}\right) C_{a} \\
0 & \left(m_{a} l_{g a}+m_{o} l_{o}\right) C_{a}
\end{array}\right]\left[\begin{array}{c}
C \theta_{b} \\
C \theta_{b a}
\end{array}\right] \mathbf{g}} \\
-\left[\begin{array}{cc}
m_{b} l_{g b} S_{b} & \left(m_{a} l_{g a}+m_{o} l_{o}\right)_{a} \\
0 & \left(m_{a} l_{g a}+m_{o} l_{o}\right) S_{a}
\end{array}\right]\left[\begin{array}{l}
S \theta_{b} \\
S \theta_{b a}
\end{array}\right] \mathbf{g}
\end{gathered}
$$

Thus, the following equations can be used to obtain $m_{o}$

$$
\begin{aligned}
& \tau_{a}=\left(m_{a} l_{g a}+m_{o} l_{\mathrm{g} o}\right) C_{a} C \theta_{b a} \mathrm{~g}- \\
& \left(m_{a} l_{g a}+m_{o} l_{g o}\right) S_{a} S \theta_{b a} \mathrm{~g} \\
& \tau_{b}=\left(m_{b} l_{g b} C_{b}+m_{a} l_{b}+m_{o} l_{o}\right) C \theta_{b} \mathbf{g}- \\
& m_{b} l_{g b} S_{b} S \theta_{b} \mathbf{g}-\left(m_{a} l_{g a}+m_{o} l_{o}\right) S_{a b} S \theta_{b a} \mathbf{g}
\end{aligned}
$$

where $\mathrm{Lgo}_{\mathrm{g}}$ is the distance from the centre of the object to the swing, determined using the Digiclops system.

$$
m_{o}=\frac{\tau_{a}-m_{a} l_{g a} \mathrm{~g}\left(C_{a} C \theta_{b a}-S_{a} S \theta_{b a}\right)}{l_{g a} \mathrm{~g}\left(C_{a} C \theta_{b a}-S_{a} S \theta_{b a}\right)}=m_{o 1}
$$

$$
\begin{gathered}
m_{o}=\frac{\tau_{b}-\left(m_{b} l_{g b} C_{b}+m_{a} l_{b}\right) C \theta_{b} \mathbf{g +}}{l_{o} \mathbf{g}\left(C \theta_{b}-S_{a b} S \theta_{b a}\right)} \\
\underline{m_{b} l_{g b} S S_{b} S \theta_{b} \mathbf{g}+m_{a} l_{g d} S_{a b} S \theta_{b a} \mathbf{g}}=m_{o 2}
\end{gathered}
$$

Although $\mathrm{m}_{\mathrm{o}}$ can be obtained using Eqs. (39) and (40), the two equations are slightly different. Eq. (39) is relatively simple and must thus be used when possible. However, when the arm is vertical to the ground, singularity appears, and $\tau_{\mathrm{a}}$ becomes extremely small. In this case, it is difficult to obtain the force. Therefore, the following formula is used to define $\mathrm{m}_{\mathrm{o}}$

$$
m_{o}=\left\{\begin{array}{l}
\mathrm{m}_{\mathrm{o} 1}\left|\theta_{a b}\right|<20^{\circ} \\
\mathrm{m}_{\mathrm{o} 2} \quad \text { else }
\end{array}\right.
$$

Using $\mathrm{m}_{0}$, a rough initial value can be obtained through the following equation:

$$
I_{o 1}=\rho \int_{l_{\text {min }}}^{l_{\text {max }}} V_{1} d l
$$

where $1_{\text {omin }}$ and $1_{\text {omax }}$ denote the minimum and maximum distances of the object from the swing, respectively. The object is expected to be evenly distributed, and volume $\mathrm{V}_{1}$ is determined through the vision information. $\mathrm{I}_{\mathrm{o} 1}$ can be used as the initial value of the parameter identification.

\section{Simulation experiment}

To verify the effect of the proposed scheme, the following simulation experiments are performed over the MATLAB Simulink platform. Three methods: compound 
PID control (CPID), common sliding mode control (SM) and the proposed strategy, namely, sliding mode control with initial value (SMI), as shown in Figure 7, are used in the comparison experiments.

The schematics of the controllers are shown in the following figures.

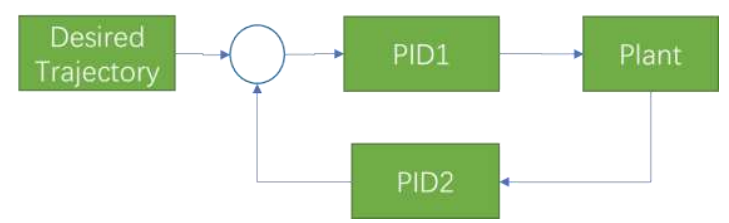

Figure 5 Compound PID controller (CPID)

The CPID controller is shown in Figure 5. This system represents a practical controller in a linear system. The zero and pole points are optimized through the feedforward and feedback PID controller, and the PID parameters can be adjusted in conjunction with the Routh stability criterion.

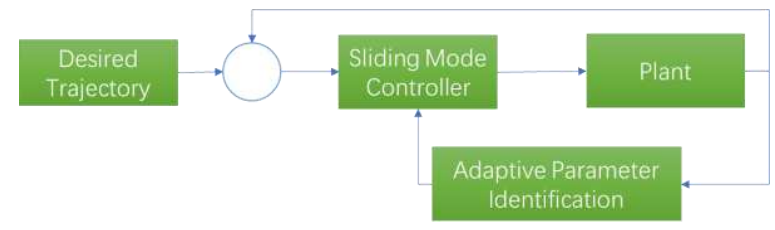

Figure 6 Sliding mode controller (SM)

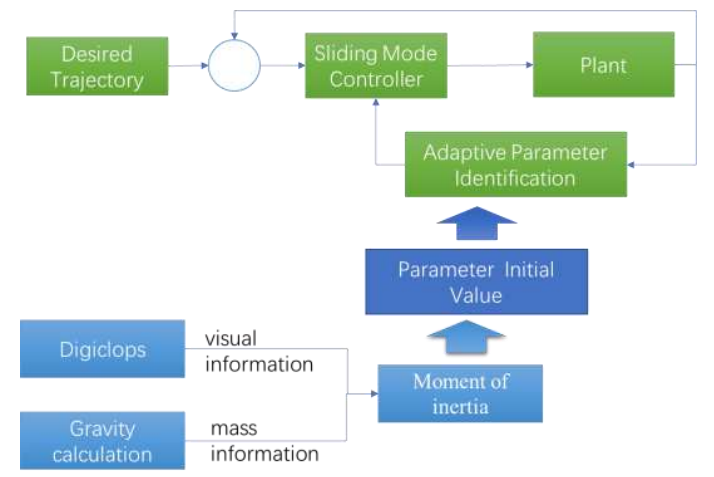

Figure 7 Sliding mode controller with initial value identification (SMI)

The sliding mode controller is shown in Figure 6. The input modules in Figures 4 and 5 function on the desired trajectory output, such as the position, velocity and acceleration. The trajectory is $\mathrm{y}=\sin (\pi \mathrm{t})$.

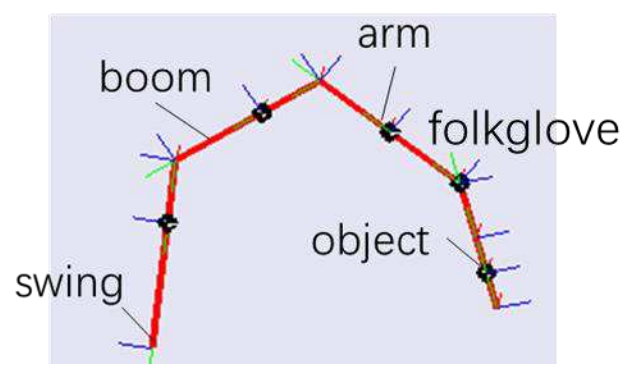

Figure 8 SimMechanics plant

The plant built by SimMechanics (Figure 8) is applied in the simulation program. As shown in Figure 6, the load features can be adjusted by setting the mass and gravity centre. Considering the work scenario, the load parameter is a constant value after it is determined, and no disturbance force is applied. Only the commonly used friction force based on the LuGre model is introduced.

$D=v_{0} z+v_{1} \frac{d z}{d t}+v_{2} \&$

where $\mathrm{z}$ is the average deformation of the bristles, $v_{0}$ is the bristle stiffness coefficient, $v_{1}$ is the microdamping coefficient, and $v_{2}$ is the viscous coefficient.

The parameters adopted in the program are listed in Table I.

Table I Parameters in the simulation program

\begin{tabular}{|c|c|c|c|}
\hline Parameter & Description & Value & Unit \\
\hline$\overline{A_{s}}$ & Area of the piston & $6.08 \times 10^{3}$ & $\mathrm{~m}^{2}$ \\
\hline$\beta_{\mathrm{e}}$ & $\begin{array}{l}\text { Bulk modulus of the } \\
\text { fluid }\end{array}$ & 690 & $\mathrm{MPa}$ \\
\hline $\mathrm{w}$ & $\begin{array}{lll}\text { Servo } & \text { valve } & \text { area } \\
\text { gradient } & & \end{array}$ & 0.008 & $\mathrm{~m}$ \\
\hline Ps & Supply pressure & 2 & MPa \\
\hline $\mathrm{V}_{0}$ & Total fluid volume & $9.05 \times 10^{-4}$ & $\mathrm{~m}^{3}$ \\
\hline $\mathrm{C}_{\mathrm{t}}$ & $\begin{array}{l}\text { Discharge coefficient of } \\
\text { the cylinder }\end{array}$ & $7 \times 10^{-9}$ & $\mathrm{~m}^{5} /(\mathrm{N} \cdot \mathrm{s})$ \\
\hline $\mathrm{k}_{\mathrm{q}}$ & Pressure gain & $5.09 \times 10^{5}$ & $\mathrm{~m}^{4} \mathrm{~s} / \mathrm{kg}$ \\
\hline $\mathrm{k}_{\mathrm{a}}$ & Amplification gain & 1.0 & $\mathrm{~mA} / \mathrm{V}$ \\
\hline
\end{tabular}

In the simulation program, the real value of $m_{o}$ is 2681 $\mathrm{kg} \cdot \mathrm{m}^{2}$, and the calculated values of the parameters are $\sigma=\left[-4547,-0.0113,0.2984,3.73 \times 10^{-4}, 14.48\right]$ 

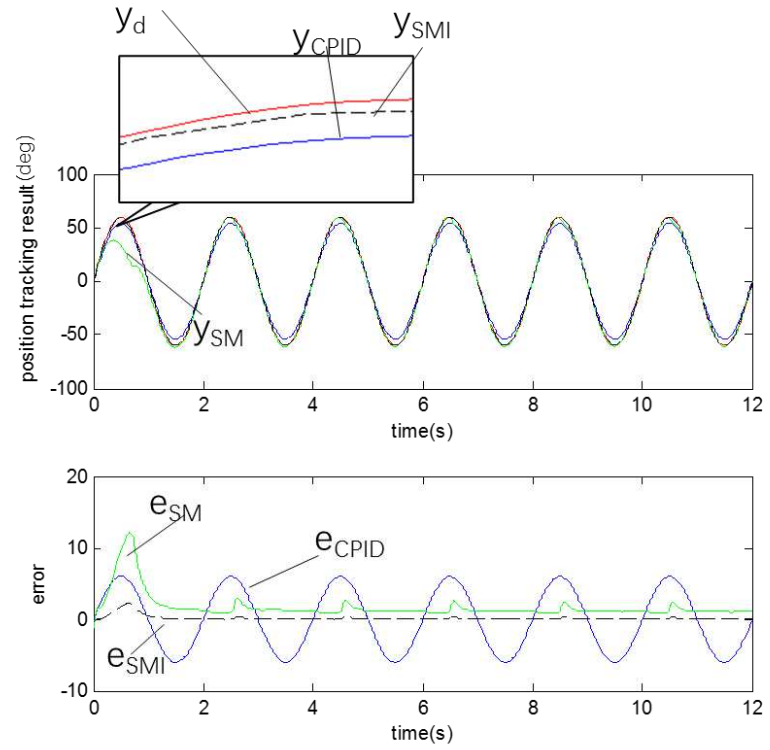

Figure 9 Trajectory tracking result

The initial parameter value is input to the adaptive parameter identification module by setting the parameters in the $\mathrm{S}$ function. To validate the performance of the initial value identification, the initial values of $M$ and $\sigma$ are set. In $\mathrm{SM}$, the initial value of $\mathrm{m}_{\mathrm{o}}$ is set as the unloading moment of inertia, $723 \mathrm{~kg} \cdot \mathrm{m}^{2}$; thus, the initial values of $\sigma$ are

$\sigma=[-16861,-0.0417,1.1065,0.0014,53.68]$

In SMI, the initial value of $\mathrm{m}_{\mathrm{o}}$ is set as $2469 \mathrm{~kg} \cdot \mathrm{m}^{2}$; thus, the initial values of $\sigma$ are

$\sigma=\left[-4937.4,-0.0122,0.3240,4.05 \times 10^{-4}, 15.718\right](47)$

The reference trajectory is $\theta_{\mathrm{d}}=60 \times \sin \pi \mathrm{t}\left({ }^{\circ}\right)$.

According to the simulation experiment results shown in Figures 9 and 10, the CPID method has a certain effect. Because the CPID does not have the parameter estimation function, the initial and final tracking errors are similar. The trajectory tracking error of the SM controller is considerably smaller than the steady-state error of the CPID. However, due to parameter coupling [14], the parameters of the SM method cannot approach the target parameters, and thus, the performance of the controller is limited. The SMI trajectory tracking results in the later stage are the best among the three methods because of the superior parameter estimation performed through the initial value. However, the parameter identification results are slightly different from the real values because of the limitations of the estimation algorithm.
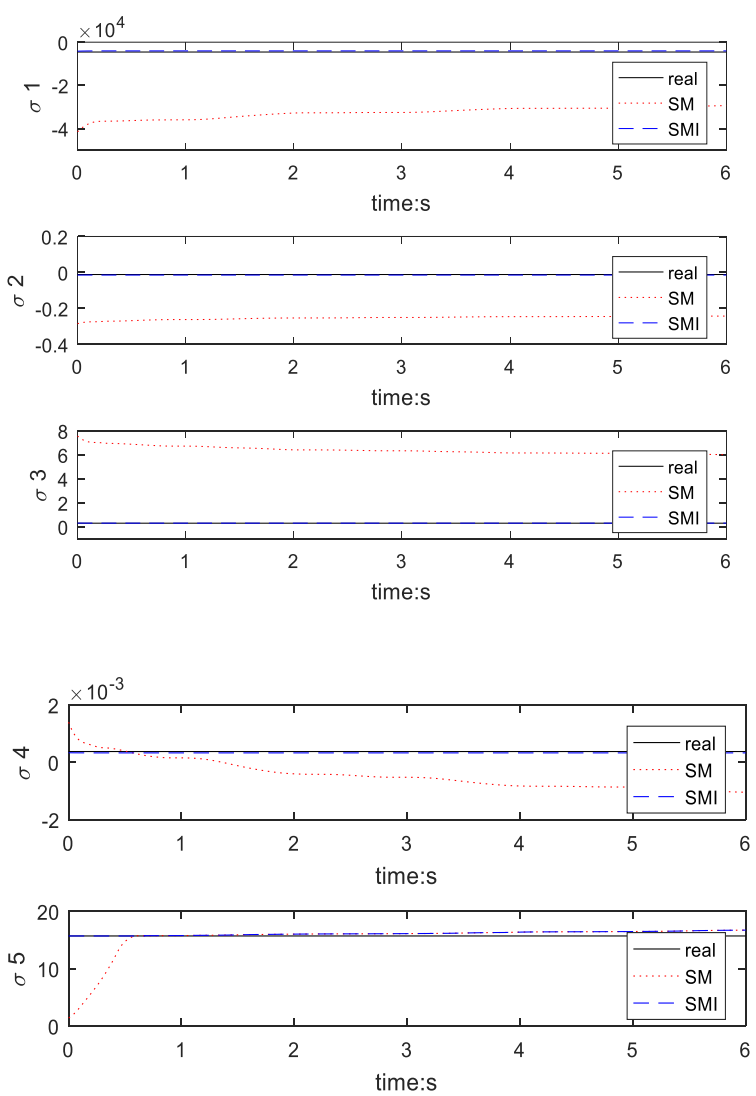

Figure 10 Parameter identification result

\section{Experiment}

This section describes the moment of inertia identification experiment and validation experiment of SMI. The system setup and implementation issues are outlined in Section 6.1. The process and result of the identification experiment are described in Section 6.2, and the control effect verification experiment is described in Section 6.3.

\subsection{Experiment setup and implementation issues}

The experimental setup consists of the following hardware components.

(1) Construction robot reconstruction for the $10 \mathrm{~T}$ LiuGong excavator.

(2) Pointgrey Research Colour Digiclops, with three 
Sony $1 / 3$ " progressive scan CCD cameras.

(3) Rexroth Huade 4 WRE16-10 servo valve.

(4) Druck PTX1400 pressure transmitters with an operating pressure range of $25 \mathrm{MPa}$.

(5) Miran LVDT20 rod-type displacement sensor.

6) ADVANTECH PCI-1710UL Data acquisition card (DAQ) with 12-bit data acquisition and $100 \mathrm{kHz}$ sampling rate.

For simplicity, the initial values are determined by offline experiments. The hardware of the control system consists of two PC-compatible computers that communicate through a local area network. All analogue measurement signals (cylinder position, chamber pressure, supply pressure and load force) are fed back to the slave PC through four plug-in DAQ cards.

\subsection{Moment of inertia identification experiment}

To test the effect of the vision information combined with the gravity recognition algorithm of moment of inertia, as described in Section 3.2, a three-dimensional vision and gravity compensation method was applied to grasp stone and wood specimens.

In the experiment, the object stone is grabbed by a folkglove at the centre position and $20 \mathrm{~cm}$ to the left. The position is manually measured to ensure accuracy. In another experiment, a wood object is grabbed.

The moment of inertia result is $I_{\text {sum }}$. According to Table II, the identification results and real results are different, especially in the case of wood objects, primarily owing to the irregular shape of the objects. The irregularity of the work object at the work site highlights the necessity of using the integrated adaptive algorithm and initial value identification algorithm.

The identification experiment results are shown in Table II.

Table II Results of identification experiments

\begin{tabular}{lll}
\hline & $\begin{array}{l}\text { Calculated } \\
\text { value }\end{array}$ & $\begin{array}{l}\text { Identified } \\
\text { value }\end{array}$ \\
\hline Stone (M) & 2681 & 2670 \\
Stone (L) & 2690 & 2710 \\
Wood (M) & 1809 & 2087 \\
Wood (L) & 1824 & 2123 \\
\hline
\end{tabular}

\subsection{Carrying experiment}

To validate the effect of the proposed control scheme in trajectory tracking, the following experiment is performed. Three controllers, CPID, SM and SMI, are used to grab a stone and apply a step desired displacement. These conditions are similar to real working conditions.

Using CPID, SM and SMI, the following processes are performed through automatic control methods:

(1) Grasp the object and lift it approximately $5 \mathrm{~cm}$.

(2) Set the parameters of SM and SMI, according to (45) and (46), respectively.

(3) Initiate data acquisition.

(4) Use the step signal to move the swing to the left for 5 $\mathrm{s}$ at the desired speed of $0.02 \mathrm{~m} / \mathrm{s}$, release the folkglove, and allow the object to land freely.

(5) Set the parameters (SM and SMI) to the unloaded initial value (45), and use the step signal to move the swing to the right for $5 \mathrm{~s}$ with a target speed of $0.02 \mathrm{~m} / \mathrm{s}$.

The evaluation indicators of the trajectory tracking results include the maximum error and root mean square error (RMSE).

The experimental process is similar to the real working conditions.

Figure 11 shows the velocity tracking result. Table III presents the trajectory tracking results. In the carrying process, the SM and SMI sliding mode algorithms are superior to the CPID due to the uncertainties related to the object. SM exhibits an inferior result owing to the convergence effect of the estimation algorithm. SMI combined with visual and gravity parameter identification exhibits an enhanced control performance. 


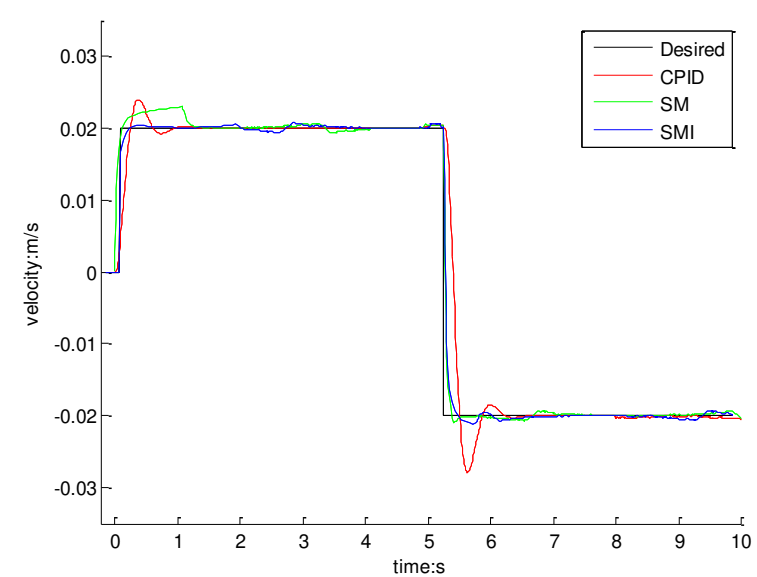

Figure 11 Velocity tracking result

Table III Trajectory tracking result

\begin{tabular}{lll}
\hline Controller & $\begin{array}{l}\text { Maximum } \\
\text { error }\end{array}$ & RMSE \\
\hline CPID & 0.004 & 0.1083 \\
SM & 0.003 & 0.0530 \\
SMI & 0.0008 & 0.0183 \\
\hline
\end{tabular}

This phenomenon occurs because the initial value is accurately set in advance. The SMI method thus exhibits a high accuracy at the initial moment. Due to the relatively brief period of the swing in each handling and unloading procedure, the working performance is mostly determined by the initial period, and SMI is thus a practical method.

Moreover, in the process of handling and unloading, due to the uncertainties associated with the object weight, it is challenging to obtain satisfactory results from the simple identification algorithm. The experimental results demonstrate the importance of the initial value identification method for all sliding mode strategies with adaptive parameter identification. In addition to trajectory tracking, this method can be used in other fields, such as energy-saving control.

\section{Conclusion}

This study focuses on the adaptive robust sliding mode control scheme for trajectory tracking of a swing with a double actuated hydraulic cylinder in a hydraulic construction robot.

A novel control scheme that obtains the initial value of the moment of inertia of swing through the robot gravity identification algorithm and stereo vision information is proposed. The scheme can be used to overcome the limitations associated with the low convergence speed of the parameter identification algorithm and large change in the inertia caused by the change in the work object.

Simulation and online experiments are performed to validate the effect of the novel scheme. The simulation experiment demonstrates the superiority of the SM algorithm over the CPID algorithm and necessity of obtaining the initial value for the sliding mode algorithm in SMI. Online experiments indicate that the moment of inertia deviation obtained using Digiclops combined with the gravity identification method is more than $20 \%$, indicating the necessity of using the parameter adaptive algorithm in the relevant strategy. Comparison experiments involving the grabbing of stones using SMI and SM show that the proposed SMI method is more accurate than the SM method and CPID control algorithm. This research can facilitate further work on robots and parameter estimation-based control.

\section{Declaration}

\section{Acknowledgements}

The authors sincerely thanks to Professor ** of ** University for his critical discussion and reading during manuscript preparation.

\section{Funding}

Supported by National Key R\&D Program of China (Grant No. 2016YFC0802900) and Jilin Provincial Natural Science Foundation of China (Grant No. 2015021117).

\section{Availability of data and materials}

The datasets supporting the conclusions of this article are included within the article.

\section{Authors' contributions}

The author' contributions are as follows: Ding-Xuan Zhao and Jing-Wei Hou were in charge of the whole trial; Jing-Wei Hou wrote the manuscript; Zhu-Xin Zhang assisted with sampling and experiment analyses. All authors reviewed the manuscript.

\section{Competing interests}

The authors declare no competing financial interests.

\section{Consent for publication}

Not applicable

\section{Ethics approval and consent to participate}

Not applicable 


\section{Reference}

[1] G.J. Maeda, Learning and Reacting With Inaccurate Prediction: Applications to Autonomous Excavation (Ph.D. thesis) School of Aerospace, Mechanical and Mechatronic Engineering, The University of Sydney, 2013.

[2] Dadhich S, Bodin U, Andersson U. Key Challenges in Automation of Earth-Moving Machines. Automation in Construction. 2016, 68, (5):212-22.

[3] Fang Y, Yang J, Chai X. Hybrid Control of Hydraulic Press Machine Based on Robust Control. Chinese Journal of Mechanical Engineering. 2008, 21(2): 72-76.

[4] Lin T, Wang Q. Hydraulic Accumulator-Motor-Generator Energy Regeneration System for A Hybrid Hydraulic Excavator. Chinese Journal of Mechanical Engineering. 2012, 25(3): 1121-1129.

[5] Jin Z, Pagilla PR, Maske H, Chowdhary G. Task Learning, Intent Prediction, and Adaptive Blended Shared Control with Application to Excavators. IEEE Transactions on Control Systems Technology. 2021, 29(3): 18-28.

[6] Feng H., Yin C., Ma W., Yue H.. Parameters Identification and Trajectory Control for A Hydraulic System. ISA Transactions. 2019, 81 (9): 228-240

[7] Kim S, Park J, Kang S, Pan YK, Kim HJ. A Robust Control Approach for Hydraulic Excavators Using $\mu$-synthesis. Int $J$ Control Autom. 2018;16(4): 615-1628.

[8] Hou J., Zhao D., Gong M. etc. Strategy-switching Control for Hydraulic Force Bilateral Servo System When Catching Objects[J]. Transactions of the Chinese Society for Agricultural Machinery. 2012, 43 (07) :190-193+211. (in Chinese)

[9] T. Chen. D. Zhao. and Z. Zhang. Research on Master Slave Robots Bilateral Control Strategy with Force Tele-Presence[C]. Proceedings of the IEEE International Conference on Automation and Logistics, ICAL 2007. Wuhan, China, September 27-29: 2301-2305.

[10] M. Pla-Castells, I. García-Fernández, and R. J. Martínez, Interactive Terrain Simulation and Force Distribution Models in Sand Piles, in Lecture Notes in Computer Science (including subseries Lecture Notes in Artificial Intelligence and Lecture Notes in Bioinformatics), Francisco, USA, September $1-5,2006$, :392-401.

[11] Bingül Zafer, Karahan Oğuzhan. A Fuzzy Logic Controller Tuned with PSO For 2 DOF Robot Trajectory Control. Expert Syst Appl
2011;38(1):1017-31.

[12] Li Q, Yuan J, Zhang B, Wang H. Artificial Potential Field Based Robust Adaptive Control for Spacecraft Rendezvous and Docking Under Motion Constraint. ISA Trans 2019,81(1):1-12.

[13] S. Li,Y. Shan,J. Yu,Y. Ke. Actuation Spaces Synthesis of Lower-Mobility Parallel Mechanisms Based on Screw Theory. Chinese Journal of Mechanical Engineering, 2021, 34:28. doi: 10.1186/s10033-021-00546-7.

[14] D. Xi , F. Gao. Type Synthesis of Walking Robot Legs. Chinese Journal of Mechanical Engineering, 2018, 31(1): 15. doi: 10.1186/s10033-018-0216-7.

[15] Peng J, Yang Z, Wang Y, Zhang F, Liu Y. Robust Adaptive Motion/Force Control Scheme for Crawler-Type Mobile Manipulator with Nonholonomic Constraint Based on Sliding Mode Control Approach. ISA Trans 2019,81(1): 1-12.

[16] Hashemi M, Shahgholian G. Distributed Robust Adaptive Control of High Order Nonlinear Multi Agent Systems. ISA Trans 2018;80(3):14-27.

[17] Zhu Z-C, Li X, Shen G, Zhu W-D. Wire Rope Tension Control of Hoisting Systems Using a Robust Nonlinear Adaptive Backstepping Control Scheme. ISA Trans 2018,80(3):256-72.

[18] Izadbakhsh A, Khorashadizadeh S, Ghandali S. Robust Adaptive Impedance Control of Robot Manipulators Using SzÁSz-Mirakyan Operator as Universal Approximator. ISA Transactions. 2020, 82(6): 1-11.

[19] Wang C, Jiao Z, Quan L. Nonlinear Robust Dual-Loop Control for Electro-Hydraulic Load Simulator. ISA Transactions. 2015, 77(3): 280-289.

[20] Zhang J, Lu Z, Xu B, Su Q. Investigation on The Dynamic Characteristics and Control Accuracy of a Novel Proportional Directional Valve with Independently Controlled Pilot Stage. ISA Transactions. 2019, 81(5): 218-230.

[21] Gao Y, Jin Y, Zhang Q. Motion Planning Based Coordinated Control for Hydraulic Excavators. Chinese Journal of Mechanical Engineering. 2009, 22(2) 97-101.

[22] Chegini S, Yarahmadi M. Quantum sliding mode control via error sliding surface. Journal of Vibration and Control. 2018, 24, 5345-52.

[23] Das M, Mahanta C. Optimal second order sliding mode control for nonlinear uncertain systems. Isa Transactions. 2014, 53, 1191-8.

[24] Incremona GP, Rubagotti M, Ferrara A. Sliding Mode Control of 
Constrained Nonlinear Systems. Ieee Transactions on Automatic

Control. 2017, 62, 2965-72.

[25] Chen YY, Chen YH, Huang CY. Wheeled mobile robot design with robustness properties. Advances in Mechanical Engineering. 2018, (10):944-953.

[26] Yue F, Li X. Robust adaptive integral backstepping control for optoelectronic tracking system based on modified LuGre friction model. ISA Trans 2018(8):312-21.

[27] Mohanty A, Yao B. Indirect Adaptive Robust Control of Hydraulic Manipulators with Accurate Parameter Estimates. IEEE Transactions on Control Systems Technology. 2011, 19(3): 567-75.

[28] J.B Cao, B.G,Cao. Neural Network Sliding Mode Control Based on On-line Identification for Electric Vehicle With ultra capac1tor-battcD hybrid power. International Journal of Control, Automation and systems, 2009,7(3):209-218

[29] Luo RC, Perng YW. Adaptive Skew Force Free Model-Based Synchronous Control and Tool Center Point Calibration for a Hybrid 6-DoF Gantry-Robot Machine. IEEE-Asme Transactions on Mechatronics. 2020, 25(4): 964-76.

[30] Wang F, He L. FPGA-Based Predictive Speed Control for PMSM System Using Integral Sliding-Mode Disturbance Observer. IEEE Transactions on Industrial Electronics. 2021, 68(8): 972-81.

[31] C J Yang, B Niu, J F Zhang, et al. Different Structure Based Control System of The Puma Manipulator with An Arm Exoskeleton. Proceedings of the IEEE Conference on Robotics, Automation and Mechatronics, Singapore, December 12-15, 2004: 572-577.

[32] S Kaitwanidvilai, M Parnichkun. Force Control in A Pneumatic System Using Hybrid Adaptive Neuro-Fuzzy Model Reference Control. Mechatroincs, 2005, 15(1): 23-41.

[33] Wang S, Na J, Xing Y. Adaptive Optimal Parameter Estimation and Control of Servo Mechanisms: Theory and Experiments. IEEE Transactions on Industrial Electronics. 2021, 68(3): 598-608.

[34] Na J, Xing Y, Costa-Castello R. Adaptive Estimation of Time-Varying Parameters with Application to Roto-Magnet Plant. IEEE Transactions on Systems Man Cybernetics-Systems. 2021, 51(7): 731-41.

[35] Chen G, Jin B. POSITION-POSTURE TRAJECTORY TRACKING OF A SIX-LEGGED WALKING ROBOT. International Journal of Robotics \& Automation. 2019, 34, 24-37.
[36] Chen YY, Chen YH, Huang CY. Wheeled mobile robot design with robustness properties. Advances in Mechanical Engineering. $2018,10$.

[37] Liu X, Wang W, Li X, Liu F, He Z, Yao Y, et al. MPC-based high-speed trajectory tracking for 4WIS robot. ISA transactions. 2021.

[38] Spyrakos-Papastavridis E, Dai JS. Minimally Model-Based Trajectory Tracking and Variable Impedance Control of Flexible-Joint Robots. Ieee Transactions on Industrial Electronics. 2021, 68, 6031-41.

[39] H.Kato, H.Yamada, T.Muto. Master-Slave Control for a Tele-Operation System of Construction Robot, Second report: Expansion to an articulated arm by gravity compensation. Proceedings of the Japan Fluid Power Systems Society, 200334 (4):85-91.

[40] Pi Y, Wang X. Trajectory Tracking Control of a 6-DOF Hydraulic Parallel Robot Manipulator with Uncertain Load Disturbances. Control Engineering Practice. 2011, 19(2): 185-193.

\section{Biographical notes}

Jing-Wei Hou, born in 1978, is currently a PhD at School of mechanical and aerospace engineering, Jilin University, China. He received his $\mathrm{PhD}$ degree in Jilin University, China, in 2008. His research interests include engineering robot and hydraulic servo control.

Tel: +86-13504316084; E-mail: houjw@jlu.edu.cn

Ding-Xuan Zhao, born in 1965, is currently a professor at School of mechanical engineering YanShan University, China. He received his PhD degree in Jilin University, China, in 1992.

E-mail: zdx@ysu.edu.cn

Zhu-Xin Zhang, born in 1967, is currently a professor at School of mechanical engineering YanShan University, China. He received his PhD degree in Jilin University, China, in 2007. 


\section{Supplementary Files}

This is a list of supplementary files associated with this preprint. Click to download.

- Supplementaryfile.docx 